\title{
BMJ Open Evaluating the provision of Further Enabling Care at Home (FECH+) for informal caregivers of older adults discharged home from hospital: protocol for a multicentre randomised controlled trial
}

Anne-Marie Hill (D) , ${ }^{1,2}$ Rachael Moorin (D) , ${ }^{3}$ Susan Slatyer, ${ }^{4}$ Christina Bryant, ${ }^{5}$ Keith Hill, ${ }^{6}$ Nicholas Waldron, ${ }^{7}$ Samar Aoun, ${ }^{8,9}$ Ami Kamdar, ${ }^{10}$ Laurie Grealish, ${ }^{11,12}$ Caroline Reberger, ${ }^{13}$ Cindy Jones, ${ }^{14,15}$ Mary Bronson, ${ }^{16}$ Max K Bulsara (D) ,17 Sean Maher, ${ }^{13}$ Tracey Claverie, ${ }^{11}$ Wendy Moyle ${ }^{12,14}$

To cite: Hill A-M, Moorin R, Slatyer S, et al. Evaluating the provision of Further Enabling Care at Home ( $\mathrm{FECH}+$ ) for informal caregivers of older adults discharged home from hospital: protocol for a multicentre randomised controlled trial. BMJ Open 2021;11:e046600. doi:10.1136/ bmjopen-2020-046600

- Prepublication history and additional supplemental material for this paper are available online. To view these files, please visit the journal online (http://dx.doi.org/10.1136/ bmjopen-2020-046600).

Received 04 November 2020 Accepted 25 May 2021

Check for updates

(C) Author(s) (or their employer(s)) 2021. Re-use permitted under CC BY-NC. No commercial re-use. See rights and permissions. Published by BMJ.

For numbered affiliations see end of article.

Correspondence to

Dr Anne-Marie Hill;

Anne-Marie.Hill@curtin.edu.au

\section{ABSTRACT}

Introduction There are personal and societal benefits from caregiving; however, caregiving can jeopardise caregivers' health. The Further Enabling Care at Home $(\mathrm{FECH}+)$ programme provides structured nurse support, through telephone outreach, to informal caregivers of older adults following discharge from acute hospital care to home. The trial aims to evaluate the efficacy of the $\mathrm{FECH}+$ programme on caregivers' health-related quality of life (HRQOL) after care recipients' hospital discharge.

Methods and analysis A multisite, parallel-group, randomised controlled trial with blinded baseline and outcome assessment and intention-to-treat analysis, adhering to Consolidated Standards of Reporting Trials guidelines will be conducted. Participants ( $\mathrm{N}=925$ dyads) comprising informal home caregiver (18 years or older) and care recipient (70 years or older) will be recruited when the care recipient is discharged from hospital. Caregivers of patients discharged from wards in three hospitals in Australia (one in Western Australia and two in Queensland) are eligible for inclusion. Participants will be randomly assigned to one of the two groups. The intervention group receive the $\mathrm{FECH}+$ programme, which provides structured support and problem-solving for the caregiver after the care recipient's discharge, in addition to usual care. The control group receives usual care. The programme is delivered by a registered nurse and comprises six 30-45 min telephone support sessions over 6 months. The primary outcome is caregivers' HRQOL measured using the Assessment of Quality of Life-eight dimensions. Secondary outcomes include caregiver preparedness, strain and distress and use of healthcare services. Changes in HRQOL between groups will be compared using a mixed regression model that accounts for the correlation between repeated measurements. Ethics and dissemination Participants will provide written informed consent. Ethics approvals have been obtained from Sir Charles Gairdner and Osborne Park Health Care Group, Curtin University, Griffith University,
Strengths and limitations of this study

- The study uses a multicentre randomised design with blinded baseline and outcome assessment.

- Follow-up time points of 6 and 12 months allow robust evaluation of the effect of the Further Enabling Care at Home+ programme on caregivers' outcomes as well as the use of health services.

- Evaluating secondary outcomes, including caregiver distress and preparedness to care will provide further insight into the intervention effect.

- Participants cannot be blinded to receiving the intervention.

- The care recipients are older and therefore mortality and hospital readmission may affect the recruitment or retention of participants.

Gold Coast Health Service and government health data linkage services. Findings will be disseminated through presentations, peer-reviewed journals and conferences. Trial registration number ACTRN12620000060943.

\section{INTRODUCTION}

In Australia, an estimated 2.65 million people provide informal care, $32 \%$ as primary caregivers, of whom over $60 \%$ provide support for a spouse or parent. ${ }^{1}$ The proportion of older adults (aged 65 years and over) across the world is expected to double from $12 \%$ in 2020 to $22 \%$ by $2050^{2}$ and in Australia older people expect to continue to live at home, with less than $5 \%$ of older people living in Residential Aged Care (RAC) accommodation. ${ }^{1}$ Hence informal caregivers are critical in supporting older people to live at home for as long as possible. ${ }^{3}$ 
Caregivers, however, report significant personal costs associated with care, including serious financial, social and health problems. ${ }^{45}$ Caregivers repeatedly report lower levels of well-being than the general population. Mental health risks are particularly severe, with caregivers reporting significantly higher levels of loneliness, anxiety, depression and stress than the general population. ${ }^{4-7} \mathrm{~A}$ systematic review found evidence of a negative impact of caregiving on both the mental and physical health of the informal caregiver. ${ }^{8}$ Rising levels of caregiver stress are known to predict premature admission of the care recipient to RAC. ${ }^{9}$

There is limited evidence that providing supportive programmes, such as face-to-face training, telephone support or online digital programmes, can reduce the adverse consequences of caregiving for older adults. ${ }^{10-13}$ In addition to problems faced by caregivers in providing ongoing care, there is limited evidence about how to effectively support caregivers of older adults when they are discharged from hospital, including what interventions can sustain and improve caregivers' health and well-being. ${ }^{14-16}$ Older adults discharged from hospital are at high risk of functional decline, unplanned hospital readmissions and injurious falls. ${ }^{17-19}$ This transition can be particularly problematic for older adults who receive care support and their caregivers. Lack of continuity of care and inadequate communication and discharge plans during this time of transition can increase caregiver burden even further. ${ }^{20}{ }^{21} \mathrm{~A}$ recent meta-analysis of 23 trials found low quality evidence that informal caregiver interventions provided after hospital discharge may reduce caregiver burden and anxiety in stroke populations. However, they did not change health-related quality of life (HRQOL), anxiety or health resource use. ${ }^{15}$ Over $90 \%$ of the included trials were conducted in stroke populations thereby providing limited generalisability. Another systematic review of 21 trials found that telephone interventions providing support for caregivers may slightly reduce anxiety and improve preparedness to care, but did not significantly improve other caregiver outcomes. ${ }^{16}$ None of the included trials in this review measured changes in $\mathrm{HRQOL}^{16}$ and both reviews recommended further high-quality trials in caregiver populations. Recent qualitative research conducted with caregivers of older adults discharged from hospital indicated that negative impacts of caregiving at this time can stem from feelings of uncertainty exacerbated by gaps in formal support, the strain of balancing caregiving with other life demands and a sense of helplessness. ${ }^{22}$ Therefore further trials that design and evaluate interventions to support caregivers when the older adult they care for is transitioning from hospital to home are required.

We conducted a randomised controlled trial (RCT) evaluating the Further Enabling Care at Home (FECH) programme, a telephone-based intervention delivered to caregivers when the older adult they cared for was discharged from hospital. ${ }^{11}$ Telephone interventions are part of a suite of expanding telehealth technologies that demonstrate early evidence for providing support for caregivers and families. ${ }^{23} \mathrm{~A}$ brief intervention of seven Cognitive Behavioural Therapy (CBT) $60 \mathrm{~min}$ telephone sessions for caregivers of adults with dementia resulted in improved caregiver emotional well-being, decreased exhaustion and reduced depressive symptoms. ${ }^{24}$ Caregivers asked to rate their preferences for telehealth technologies rated telephone as the highest preferred form of technology compared with videoconferencing, Facebook, email and other technologies and telephones have the advantage of being usable for those without access to suitable technology and internet. ${ }^{25} \mathrm{~A}$ review of telephone and computer-delivered interventions for caregivers of people living with dementia found that these intervention have potential to improve caregiver well-being, in particular those that incorporated various elements of psycho-education, peer support, skills training and health assessments but that further high quality trials were required..$^{26}$ The FECH telephone programme provided support for caregivers in a timely and convenient manner in their own home. It was tailored to address the caregivers' identified support needs by providing immediate information and resources. ${ }^{11}$ In contrast to previous studies providing caregiver support, ${ }^{15}{ }^{16}$ the FECH programme delivered promising results when piloted with caregivers of older patients following hospital discharge. The programme significantly reduced caregiver strain and distress and increased preparedness to care. ${ }^{11}$ Caregivers also reported high levels of satisfaction with the programme. ${ }^{27}$ While the programme achieved a moderate effect size that was clinically significant, ${ }^{11}$ the FECH programme had some limitations. Caregiver support was limited to receiving three telephone contacts over 3 weeks after discharge and did not take an approach that assisted caregivers to develop and implement problem-solving skills. The trial was conducted in a single hospital ward and 141 dyads provided data at all time points. Since it is evident that caring frequently results in a significant negative impact across mental, physical, social and emotional health of caregivers, ${ }^{45}$ we seek to measure the impact of the intervention by using a HRQOL tool. These tools are patient-reported outcome measures and therefore capture the individuals own perception of their health and well-being in a broad sense including their quality of life. ${ }^{28}$ Therefore we aim to increase the duration and intensity of the programme $(\mathrm{FECH}+)$ and conduct a multicentre RCT to evaluate whether the $\mathrm{FECH}+$ programme may improve caregivers' HRQOL. Secondary aims will assist to explore potential causal mechanisms (such as reduction in caregiver distress and improved caregiver self-efficacy) by which participation in the $\mathrm{FECH}+$ programme in addition to usual discharge care may improve caregiver HRQOL. Since the original FECH programme improved caregiving preparedness, we also seek to 
examine if the $\mathrm{FECH}+$ programme results in improvements in care, and therefore in care recipients' levels of independence and symptoms of distress.

\section{Aims and hypotheses}

The primary aims of the trial are to (i) evaluate the efficacy of participation in the FECH+ programme in addition to usual discharge care on HRQOL of caregivers of older adults discharged home from hospital, compared with receiving usual discharge care alone: (ii) evaluate the cost-effectiveness of the intervention using the framework of a within-trial, cost-utility analysis. The secondary aims are to evaluate the efficacy of participation in the $\mathrm{FECH}+$ programme in addition to usual discharge care on: (i) caregiver preparedness to care, caregiver selfefficacy and levels of strain and distress; (ii) care recipient outcomes of symptoms of distress and level of independence, compared with usual discharge care alone.

The primary hypothesis is that caregivers participating in the $\mathrm{FECH}+$ programme in addition to receiving usual hospital discharge care (intervention group) when compared with caregivers receiving usual discharge care alone (control group), will report improved HRQOL. Secondary hypotheses are that the: (1) intervention group will report improved caregiving preparedness, decreased strain and distress and improved caregiver self-efficacy compared with the control group; and (2) care recipients in the intervention group will experience decreased symptom distress and improved independence compared with the control group.

A separate process evaluation will be conducted to explore how caregiving is impacted by participating in the FECH+ programmeand identify aspects of implementation that particularly contribute to effectiveness. A subsequent trial-based economic evaluation will evaluate the cost-effectiveness of the intervention using the framework of a within-trial, cost-utility analysis.

\section{METHODS AND ANALYSIS \\ Design}

The trial is a multicentre parallel two-group RCT with 1:1 dyad allocation to the intervention (FECH+ programme in addition to usual care) group or the control (usual care alone) group. The study will adhere to the Consolidated Standards of Reporting Trials guidelines (see figure 1). ${ }^{29}$ This protocol is reported in accordance with the Standard Protocol Items: Recommendations for Interventional Trials 2013 statement (see online supplemental file 1). ${ }^{30}$ The trial is registered through the Australian New Zealand Clinical Trials Registry.

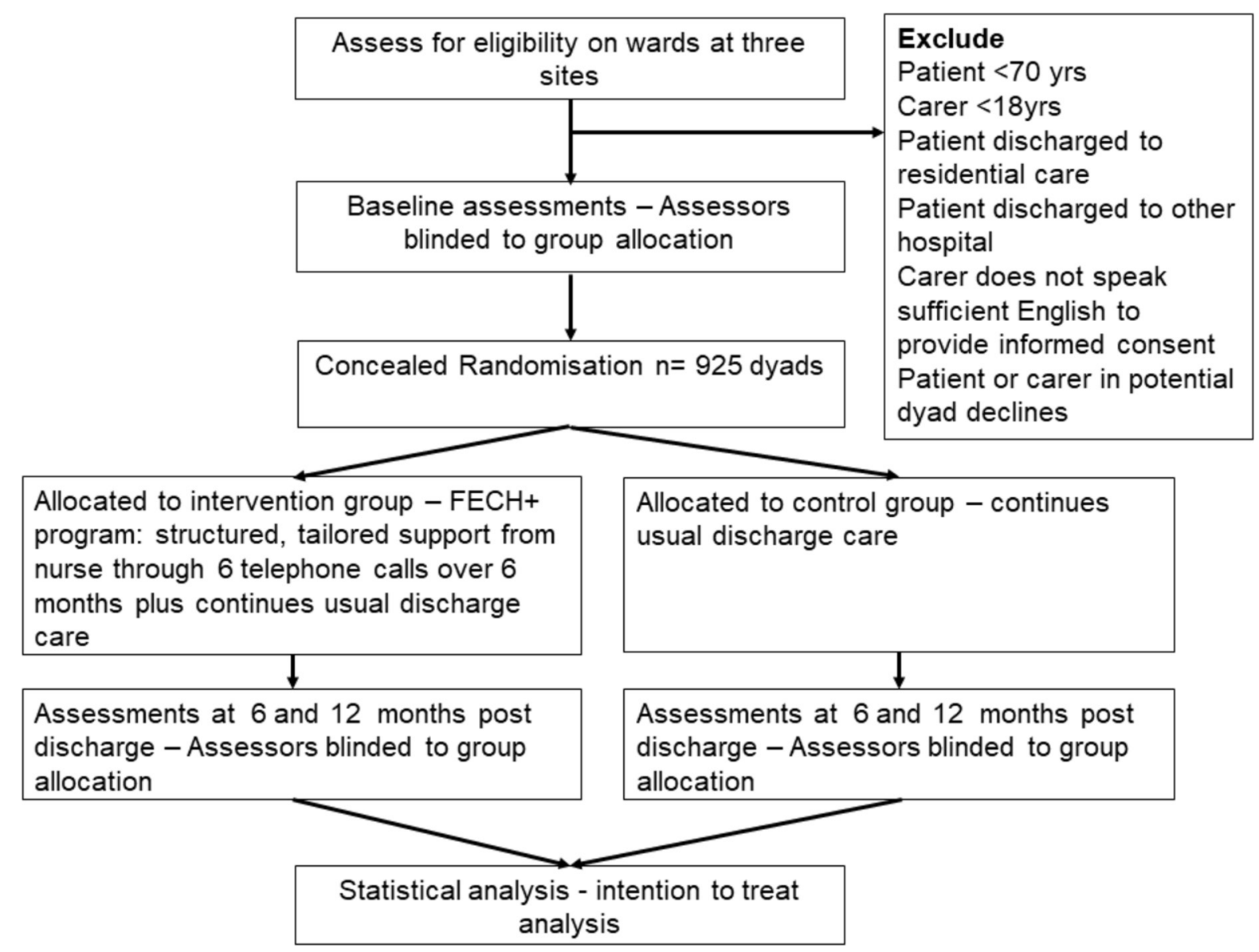

Figure 1 Participant flow through the study. FECH+, Further Enabling Care at Home. 


\section{Participants}

Participants will be enrolled as dyads (caregiver and care recipient). To be eligible for the study caregivers must be: (a) aged 18 years or older; (b) providing unpaid support as a caregiver to a patient (care recipient) aged 70 years or older when this care recipient is discharged home from a ward included in the study; care recipients must be: (c) discharged home from a ward included in the study. Exclusion criteria are that care recipients are discharged to a setting other than home (such as to a nursing home or another hospital) or undertake a hospital in the home programme. Based on a definition of a caregiver used in an Australian study investigating the support of frail older people ${ }^{31}$ caregivers are defined as family members or friends providing care and support to older people. The support must be regular (at least weekly) ongoing, homebased and can be physical and/or emotional care. In Australia unpaid care and support is provided by family and friends on a voluntary basis as compared with paid caregivers who provide personal and home care services through aged care organisations.

\section{Setting}

The trial will be conducted at three public hospitals in Australia. In Western Australia (WA), the site is Sir Charles Gairdner Hospital, a metropolitan tertiary hospital with approximately 600 beds. In Queensland (QLD), the two hospitals are Robina Hospital (approximately 400 beds) and Gold Coast University Hospital (approximately 600 beds). Wards included in the trial admit medical patients, of whom large numbers are aged over 70 years.

\section{Randomisation and blinding}

The WA Health Translation Network's Clinical Trials and Data Management Centre (CTDMC) will administer the randomisation process. The allocation list has been generated prior to trial commencement by the CTDMC using computer-generated random numbers and organised such that recruitment to the two study arms (WA and QLD) occurs at an approximately equal rate. The treatment allocation list is stored as a password protected file at Curtin University and is only accessible to the trial's CTDMC administrators. The research assistants (RAs) enter all baseline data directly into a secure online database. Completion of baseline data entry automatically triggers the allocation of the dyad (caregiver and care recipient) to the next number in the sequence and triggers an alert to the project manager, who contacts the $\mathrm{FECH}+$ nurse if the dyad is allocated to the intervention group.

The investigators on the trial team are not involved in recruitment or data collection and all investigators, including the statistical team, are blinded to group allocation until after analyses are completed. RAs who enrol patients and conduct baseline and outcome assessments are blinded to group allocation throughout the study. The project manager is the only team member to see the group allocation as she manages the trial procedure. The nurses who deliver the intervention know which participants receive the intervention, but are not involved in baseline or outcome data collection. Hospital staff who organise discharge services remain blinded to participants' enrolment into the study. Participants are not specifically informed of their group allocation but cannot be blinded to the intervention they receive. Participants will be instructed at enrolment and during monthly phone calls not to divulge their allocation to research staff. RAs who conduct baseline and outcome assessments are based in the hospitals while the nurses who deliver the intervention are located at the universities, to maintain blinding of staff.

\section{Intervention}

Participants allocated to the intervention receive the $\mathrm{FECH}+$ programme in addition to usual care. A summary of the FECH+ programme is presented in table 1 using the Template for Intervention Description and Replication checklist. ${ }^{32}$ The FECH+ programme is a telephonebased, post-hospital-discharge intervention delivered to the caregiver by one or more specially trained 'FECH+' nurses. These registered nurses have acute gerontological nursing care experience, have substantial knowledge on how to navigate the home care system and receive training in the FECH+ intervention.

The original FECH programme ${ }^{11}$ has been expanded $(\mathrm{FECH}+)$ to encourage and build the caregiver's use of problem-solving skills, through instruction and role modelling, that they can continue to use after the intervention period. Problem-solving is a practical step-by-step approach typically involving identifying and defining the problem, understanding it, setting goals and generating solutions, implementing a course of action, and evaluating its efficacy. ${ }^{33}$

\section{Training}

The FECH+ nurses undertake 3 days face-to-face training in a group setting. This includes how to assess the caregiver's understanding of discharge information, how to use the Caregiver Support Needs Assessment Tool $(\text { CSNAT })^{34}$ to facilitate the caregivers' identifying and prioritising support needs, and how to assist the caregiver to undertake a problem-solving approach. ${ }^{33}$ The CSNAT has 14 items with Likert-type response options that rate needs for support in two domains: enabling the caregiver to care for the care recipient at home and enabling support for the caregiver in their caring role. ${ }^{34}$ Training materials include the resources associated with the online CSNAT toolkit ${ }^{35}$ and an electronic manual that provides information for caregivers related to care provision for older people in Australia, as well as a manual tailored for each state that outlines the problem-solving approach. ${ }^{27}$ It is envisaged that two to three nurses will be employed at each site. Regular meetings for nurses to consolidate training procedures and to assist to monitor fidelity of intervention delivery will be conducted by the project manager and trial leaders (A-MH, CB, SS and LG). The 
Table 1 Summary of the FECH+ programme (Template for Intervention Description and Replication checklist)

(1) Brief name $\quad$ Further Enabling Care at Home $(\mathrm{FECH}+)$ for informal caregivers of older adults discharged home from hospital.

(2) Why The FECH+ programme offers a problem-solving, caregiver-focused approach to improve outcomes for the caregiver and care recipient that is complementary to usual discharge care. It is designed to provide caregivers with timely health professional support and training to use the resources available in the community. It aims to develop problem-solving skills and address the caregiver's identified needs.

(3) What-materials The caregiver completes the CSNAT ${ }^{34}$ with support provided by the FECH+ nurse. A standard operating procedures manual is used by the nurse delivering the intervention. Resources relevant for individual caregivers, such as contacts for organisations, are emailed or mailed to participants as required. Caregivers are provided with an initial booklet and an individualised summary sheet after the final phone call.

\begin{tabular}{|c|c|}
\hline $\begin{array}{l}\text { (4) What- } \\
\text { procedures }\end{array}$ & $\begin{array}{l}\text { The FECH+ nurse facilitates caregivers to (a) reflect on the current caregiving situation, (b) identify and } \\
\text { prioritise new or ongoing support needs and (c) implement a problem-solving approach to address } \\
\text { these support needs. Caregivers are guided to address three prioritised needs using problem-solving } \\
\text { techniques and goal setting. The first phone call explores the caregiver's understanding of discharge } \\
\text { information. During subsequent phone calls caring responsibilities are discussed, using the CSNAT }{ }^{34} \text { to }^{\text {th }} \\
\text { identify problems. The programme aims to facilitate the development of caregivers' problem-solving skills } \\
\text { to continue without support from the FECH+ nurse after the intervention is completed. Each contact point } \\
\text { provides an opportunity to reinforce the problem-solving skills learnt. }\end{array}$ \\
\hline
\end{tabular}

(5) Who provided Registered nurses experienced in gerontological nursing and who have received training in delivering the $\mathrm{FECH}+$ programme.

(6) How The FECH+ programme is delivered via telephone to the caregiver after the care recipient is discharged from hospital.

\section{(7) Where Delivered directly to the caregiver in their home.}

(8) When and how Six telephone calls by the FECH+ nurse after the care recipient's discharge from hospital. Call 1 during much the first week after discharge ( $15 \mathrm{~min})$; Call 2 at 2 weeks after discharge (approximately $45 \mathrm{~min})$; Calls 3 to 6 at 1, 2, 4 and 6 months, respectively, after discharge, (each approximately $30 \mathrm{~min}$ ).

(9) Tailoring

The intervention is tailored to the needs of each caregiver, using a problem-solving approach to identify, prioritise and address the top three support needs. Individual resources are provided to participants according to problems or needs identified.

(10) Modifications To be reported at study conclusion.

(11) Fidelity To be reported at study conclusion.

(12) Adherence To be reported at study conclusion.

CSNAT, Carer Support Needs Assessment Tool.

project manager will also monitor data entry for intervention delivery through the online database.

\section{Usual care}

All participants in both intervention and control groups will receive usual discharge care. Usual discharge care includes providing the care recipient and/or caregiver with a copy of the discharge letter, medications or prescriptions, outpatient appointments and home care programmes organised by the hospital team. Social work input for caregivers is not routine but may occur when prioritised by the ward social worker during admission.

\section{Outcome measures}

The primary outcome (primary aim i) is caregivers' HRQOL at 6 months after hospital discharge measured using the 35-item Assessment of Quality of Life-eight dimensions (AQoL-8D). ${ }^{36} 37$ The AQoL-8D captures psycho-social as well as physical health domains (Independent Living, Happiness, Mental Health, Coping, Relationships, Self-Worth, Pain, Senses) and can be administered by telephone. The focus on psychosocial domains makes this instrument appropriate for our study since the dominant factor affecting choice of a multi-attribute utility instrument is its ability to capture facets of health states relevant to the research question. ${ }^{38}$ It has established validity and reliability, and good psychometric properties which capture psycho-social as well as physical health domains. ${ }^{37}$ Australian norms have been established for the AQol-8D. ${ }^{39}$

Secondary outcomes are chosen to understand possible causal mechanisms of the intervention effect. For example, improved preparedness to care and reduction of caregiver strain and distress may improve caregivers HRQOL. Better prepared caregivers may in turn more effectively manage care recipients' symptoms and functional limitations. The Preparedness 
for Caregiving Scale (PCS), Family Appraisal of Caregiving Questionnaire-Palliative Care (FACQ), Symptom Assessment Scale (SAS) and Barthel Activities of Daily Living Index (BADLI) assessment instruments were previously evaluated in our pilot trial as being feasible to administer to caregivers over the telephone. ${ }^{11}$ Secondary outcomes are:

1. Caregivers' HRQOL at 12 months after hospital discharge (primary aim i: secondary time point) measured using the 35-item AQoL-8D. ${ }^{36} 37$

Other secondary outcomes evaluated at 6 and 12 months after hospital discharge include:

2. Caregivers' self-rated preparedness for caregiving (secondary aim i), measured using the PCS, ${ }^{40}$ which covers multiple domains of caregiving including preparedness to provide physical, emotional and instrumental care along with managing the stress of caregiving. This 8 -item scale has five response options $(0=$ not at all prepared, 4=verywell prepared) and is designed for use with caregivers of older adults receiving homecare/experiencing care transitions. The construct validity for the PCS has been established in older adults. ${ }^{41}$ Testing in patients with life-threatening illness confirmed satisfactory internal consistency, reliability and stability and unidimensionality. ${ }^{42}$

3. Caregivers' self-efficacy (secondary aim i), measured with the 21-item Caregiver Inventory. ${ }^{43}$ Self-efficacy is built through a mastery of tasks and ability to persist and has been shown to improve caregiver well-being. ${ }^{7}$ This questionnaire has four subscales confirmed by factor analysis: Cronbach's alpha for the scale was 0.91 in a sample of caregivers of patients for whom the main diagnoses were cancer, chronic obstructive pulmonary disease, stroke, chronic heart failure and dementia. Responses are provided using a 5-point Likert scale.

4. Caregivers' strain and distress (secondary aim i) ), measured by the corresponding subscales of the FACQ, for which good internal consistency, reliability and construct validity are confirmed. ${ }^{44}$ Responses are provided using a 5-point Likert scale.
5. Care recipients' level of independence (secondary aim ii), reported by caregivers using the BADLI, which has established reliability and validity. ${ }^{45}$

6. Care recipients' symptoms (secondary aim ii), measured using the SAS. ${ }^{46}$ Seven symptoms are each scored from 0 (not at all) to 10 (worst possible). Scores can be totalled, and caregiver proxies can complete responses. Adequate internal consistency reliability and test-retest reliability and concurrent validity have been demonstrated in older populations. ${ }^{46} 47$

Demographic data collected for caregivers and care recipients at baseline are age, gender, country of birth, number of prescription medications taken by caregiver and care recipient's length of stay in hospital. Information about the type, duration and amount of care provided by the caregiver, types of services received by the caregiver/care recipient and caregiver/care recipient health (number, type of health conditions) will also be collected.

\section{Procedure}

The study procedure is summarised in table 2. Participant dyads will be enrolled in the trial by the RAs within 24 hours of discharge from hospital. Baseline (time point 1) assessment, including demographic data collection, is completed during the first week after hospital discharge. Questionnaires are administered by phone by the RAs. Data collection at time points 2, 3 and 4 will be prompted by the project manager sending an alert to the RAs through the database and completed by phone for both intervention and control groups. This strategy ensures that FECH+ programme completion occurs prior to time point 3 data collection and maintains the RAs blinding to group allocation.

\section{Statistical analysis plan}

Characteristics of the groups will be summarised using descriptive statistics (frequencies and percentages for categorical variables; means, SD, medians and ranges for variables measured on a continuous scale). Differences in

\begin{tabular}{|c|c|c|}
\hline Time point & Time after discharge & Measurement tools administered \\
\hline T1 (baseline) data collection & $1-4$ days & AQoL-8D*, CGI, PCS, FACQ, SAS, BADLI, demographic data \\
\hline \multicolumn{3}{|c|}{ Intervention group only: FECH+ nurse contacts 1-4: 1 week, 2 weeks, 1 month, 2 months after discharge. } \\
\hline T2 data collection & 3 months & AQoL-8D, PCS, FACQ, SAS, BADLI \\
\hline \multicolumn{3}{|c|}{ Intervention group only: FECH+ nurse contacts 5-6: 4 months, 6 months after discharge. } \\
\hline T3 data collection & 6 months & $\begin{array}{l}\text { AQoL-8D, CGI, PCS, FACQ, SAS, BADLI, qualitative interview } \\
\text { (subset) }\end{array}$ \\
\hline T4 data collection & 12 months & $\begin{array}{l}\text { AQoL-8D, CGI, PCS, FACQ, SAS, BADLI, qualitative interview } \\
\text { (subset) }\end{array}$ \\
\hline
\end{tabular}

${ }^{*} A Q \circ L-8 D$ measures health-related quality of life ${ }^{36}$.

AQoL-8D, Assessment of Quality of Life-eight dimensions; BADLI, Barthel Activities of Daily Living Index; CGI, Caregiver Inventory; FACQ, Family Appraisal of Caregiving Questionnaire-palliative care; FECH+, Further Enabling Care at Home; PCS, Preparedness for Caregiving Scale; SAS, Symptom Assessment Scale. 
demographic and baseline health status variables between groups at baseline will be compared using $\chi^{2}$, t-tests or non-parametric Wilcoxon two-sample tests as appropriate. Changes from baseline in the AQoL-8D score for the caregiver will be calculated to each time point and tested for normality using the Shapiro-Wilk statistic; if not normally distributed, a Box-Cox transformation will be applied to the measure before further analysis. Comparison of the changes in AQoL-8D scores between control and intervention groups will be performed using a mixed regression model with the caregiver group identified as a random effect. This model takes into account the correlation between repeated measurements on each individual. An interaction term between time and group will be introduced into the model to test whether rates of change in the outcome differ between groups. If differences between the groups are evident at baseline, these will be included in the model as covariates so that adjustment can be made before examining differences between groups in outcomes.

In our preliminary work ${ }^{11}$ there was $<20 \%$ missing data. Missing data will be managed using multiple imputation methods informed by a sensitivity analysis to manage this, creating 25 or more data sets. ${ }^{48}$ Two analyses will be performed, namely: an analysis using only the observed data, and second, after missing value substitution, where necessary. Data will be analysed using an intention-totreat approach. Secondary outcomes will be analysed in a similar manner to the primary outcome. Statistical analyses will be conducted using Stata V.16 software (Stata Statistical Software, College Station, Texas: StataCorp). All hypothesis tests will be two-sided and $p$ values of $<0.05$ considered statistically significant.

\section{Sample size}

The primary outcome is the change in total score on the AQoL- $8 \mathrm{D}^{36}$ for the caregiver at 6 months post-discharge. A very small effect size of 0.06 has been described as being of clinical importance. ${ }^{49}$ However, we anticipate a larger effect size, based on: (a) our assessment of changes in health, measured using a health-related quality of life questionnaire [short form survey -12 item $(\mathrm{SF} 12)]^{50}$ during our preliminary study, ${ }^{11}$ in which we obtained a positive change in physical health $(0.17)$ and mental health (0.22) from baseline until immediately post-intervention; (b) that the AQoL-8D is a more appropriate outcome measure as psychosocial components of health are emphasised more; and (c) that we are now implementing an expanded intervention with longer follow-up. Therefore, we designed this study with $80 \%$ power to detect an effect size of 0.22 . This would require 648 caregiver dyads (324 in each of the control and intervention groups), determined using the $\mathrm{G}^{*}$ Power sample size calculator. ${ }^{51}$ We anticipate $30 \%$ attrition during the 12-month post-discharge period so our recruitment target to address the primary outcome variable is 925 dyads. Based on preliminary work, this sample size will also allow $80 \%$ power to detect meaningful differences in the secondary outcomes for caregiver preparedness, strain and distress, and hospitalisation costs for patients. ${ }^{11}$

\section{Process evaluation}

A process evaluation will assist in understanding the mechanism of the trial results. The process evaluation uses the framework recommended by the Medical Research Council for evaluating complex interventions. ${ }^{52}$ Caregivers' and nurses' feedback on aspects of programme implementation will be evaluated. A purposive sample (estimated 25-40) of caregivers from WA and QLD who have completed participation in the FECH+ programmewill be selected immediately after programme conclusion for inclusion in qualitative interviews. Sample selection will ensure maximum variation (eg, gender, age, relationship, caregiving duration), until data saturation. ${ }^{53}$ Qualitative, digital, audio-recorded and transcribed semi-structured telephone interviews (estimated $10-20 \mathrm{~min}$ ) will be scheduled at two time points (at the end of the intervention and 6 months later) to explore how the $\mathrm{FECH}+$ programme has influenced caregiving and caregiver experiences during and after the programme. FECH+ nurses will also be asked to record their reflections on each FECH+ programme contact. This includes identifying barriers to, or facilitators of, the effectiveness of the FECH+ programme.

Programme implementation will be examined by addressing fidelity, safety, adaptations, reach and dose. ${ }^{52}$ Data to be collected by the FECH+ nurses as they deliver the intervention include: (a) adherence to or deviation from planned $\mathrm{FECH}+$ processes including any safety concerns and how addressed; (b) information provided to caregivers and the extent to which caregivers engaged with resources provided; c) time taken to implement processes, including duration and frequency of sessions.

Qualitative data will be analysed using thematic analysis. ${ }^{53}$ Strategies to enhance the trustworthiness of findings will include verbatim transcriptions of audio-recorded interviews and an audit trail. ${ }^{53}$ Quantitative data will be presented using the framework of the process evaluation and where appropriate triangulated with qualitative data to assist in clarifying complex causal pathways. ${ }^{52}$

\section{Economic analysis}

The economic analysis plan will be published separately. Briefly, cost-effectiveness of the intervention (primary aim ii) will be measured using a within-trial cost-utility analysis. We shall evaluate the mean incremental cost and quality-adjusted life years (QALYs) according to the two randomised groups, taking an intention-to-treat perspective. A 12-month time horizon will be used, taking a health system perspective.

\section{Data management}

Data management will be overseen by a data management committee comprising a representative from the CTDMC, the WA and QLD state managers and trial leaders from WA (A-MH) and QLD (WM). The committee will undertake 
regular monthly monitoring and auditing of data entry procedures and guide all data management. Data security is primarily addressed by the use of REDCap, an online application that provides for secure data entry, storage and transfer (https://www.project-redcap.org/). The CTDMC administers REDCap and all data are stored in WA. Administrative data (which include names and dates of birth) that are accessed via the database for merging with the health data will be locked down prior to creating a merged data set and are only accessible to a CTDMC administrator. De-identified data sets will be uploaded through the university's encrypted system and stored on a password protected drive (at Curtin University). All data will be securely managed and stored at Curtin University as per National Health and Medical Research Council Australia guidelines, State data linkage services and Services Australia guidelines. Following the completion of the study analyses, a de-identified data set will be made available on reasonable request after ongoing secondary analyses are conducted and pending ethics approval from existing and requesting institutions and approval from all investigators.

\section{Trial status}

Recruitment commenced in August 2020 and is expected to be completed by approximately April 2022 with final follow-up occurring in April 2023. Primary data analyses will be completed, followed by the process evaluation. Final health data linkage will be undertaken in the 12 months after final follow-up. Health economic analyses will then be completed.

\section{Trial management}

Any amendments required to the study will be agreed on by the trial management committee consisting of all chief investigators (A-MH, WM, RM, KH, NW, SS, CB and SA), and submitted to all ethics committees for approval prior to being commenced. The trial management committee will monitor the trial in accordance with the currently approved protocol, which includes submitting annual ethics reports detailing trial progress and any adverse events to all ethics committees. Each of the named investigators on the grant shall be eligible to have authorship.

\section{Patient and public involvement}

Two consumer advocates are members of the trial team. This study was developed with caregiver input from Carers WA and the WA Consumer and Community Health Research Network, prior to submitting the application for funding. The consumer input assisted to ensure the appropriateness of the intervention and study processes. This included assisting with wording of documents for the trial and aspects of procedure and intervention delivery. The two consumer advocates are ongoing members of the trial team and continue to inform and give feedback about the study procedure. Both consumer organisations will contribute to the dissemination of results and future presentations and translation projects.

\section{Ethics and dissemination}

The study has been approved by hospitals (the Sir Charles Gairdner Osborne Park Health Care Group, Gold Coast Health Care Group) and universities (Curtin and Griffith) human research ethics committees. Approvals for linked health data for economic evaluation have been obtained from the Data Linkage Branches of WA Health, QLD Health and Services Australia (for national health administered data). All caregivers will provide written, informed consent to participate in the trial. Care recipients will also provide written, informed consent to participate in the trial. Participant information and consent forms are provided as online supplementary files (see online supplemental file 2). Cognitive impairment may occur in a care recipient who forms part of the dyad. A waiver of consent has been approved in WA for these care recipients to be included in the study. If these care recipients are encountered in QLD, we will seek consent from the appropriate substitute decision-maker.

To disseminate findings, Carers WA and Carers QLD, (two peak organisations who provide carer advocacy and support) will be asked to publicise the study completion and findings on their website. Our consumer advocates will provide advice and assistance to maximise engagement strategies through established state and national consumer networks. Papers will be published in peerreviewed journals, and abstracts submitted to relevant conferences. Practitioner and consumer forums will be held in participating hospitals and state health districts. Study participants will be provided with a summary of study findings on request.

Author affiliations

${ }^{1}$ School of Physiotherapy and Exercise Science, Curtin University, Perth, Western Australia, Australia

${ }^{2}$ School of Nursing, Midwifery and Paramedicine, Curtin University, Perth, Western Australia, Australia

${ }^{3}$ School of Public Health, Curtin University, Perth, Western Australia, Australia ${ }^{4}$ Discipline of School of Nursing, Murdoch University, Murdoch, Western Australia, Australia

${ }^{5}$ Melbourne School of Psychological Sciences, University of Melbourne, Melbourne, Victoria, Australia

${ }^{6}$ Rehabilitation Ageing and Independent Living (RAIL) Research Centre, Monash University, Melbourne, Victoria, Australia

${ }^{7}$ Armadale Health Service, Armadale, Western Australia, Australia

${ }^{8}$ School of Psychology and Public Health, La Trobe University, Melbourne, Victoria,

Australia

${ }^{9}$ Perron Institute for Neurological and Translational Science, Perth, Western Australia, Australia

${ }^{10}$ Department of General Medicine, Sir Charles Gairdner Hospital, Nedlands, Western Australia, Australia

${ }^{11}$ Gold Coast Hospital and Health Services, Gold Coast, Queensland, Australia

${ }^{12}$ School of Nursing and Midwifery Nathan and Gold Coast, Griffith University, Brisbane, Queensland, Australia

${ }^{13}$ Department of Rehabilitation and Aged Care, Sir Charles Gairdner Hospital, Nedlands, Western Australia, Australia

${ }^{14}$ Menzies Health Institute Queensland, Griffith University, Brisbane, Queensland, Australia

${ }^{15}$ Faculty of Health Sciences and Medicine, Bond University, Gold Coast, Queensland, Australia

${ }^{16}$ Medical Division, Sir Charles Gairdner Hospital, Nedlands, Western Australia, Australia 
${ }^{17}$ Institute for Health Research, The University of Notre Dame Australia, Fremantle, Western Australia, Australia

\section{Twitter Wendy Moyle @wendymoyle2}

Acknowledgements Authors thank and acknowledge the contributions of Adjunct Associate Professor Christine Toye (Curtin University) who led the trial of the original Further Enabling Care at Home programme, made a substantial contribution to the trial design, led the grant application to obtain the funding for the trial and completed ethics applications and Dr Richard Parsons who contributed to the trial design, grant application and preparation of the statistical analysis plan.

Contributors A-MH and WM led manuscript drafting. A-MH is the lead investigator on the National Health and Medical Research Council of Australia grant and all authors are named applicants on the grant. A-MH, WM, RM, KH, SS, CB, NW and $\mathrm{SA}$ contributed to trial design and will assist with monitoring trial procedure. $\mathrm{A}-\mathrm{MH}$ leads the trial with support from WM, LG and AK. CB, SS, SA, KH, LG, WM and A-MH contribute to intervention design and delivery. MKB and A-MH lead the statistical analysis plan. AK and LG lead the management of the trial sites. MB, CR, SM, TC (WA) and CJ (QLD) contribute to trial procedure, including management at the sites. RM leads the economic analyses. All authors provided critical evaluation and approval of the final submitted manuscript.

Funding This work is supported by a grant awarded by the National Health and Medical Research Council Australia (grant number APP1157834). Additional funding to support the research has been provided by Curtin University. The funders have no role in the design of the study and will not have any role in its execution, data management, analysis and interpretation nor the decision to submit results for publication. Anne-Marie Hill is supported by a National Health and Medical Research Council (Australia) Career Development Award.

Competing interests None declared.

Patient consent for publication Not required.

Provenance and peer review Not commissioned; externally peer reviewed.

Supplemental material This content has been supplied by the author(s). It has not been vetted by BMJ Publishing Group Limited (BMJ) and may not have been peer-reviewed. Any opinions or recommendations discussed are solely those of the author(s) and are not endorsed by BMJ. BMJ disclaims all liability and responsibility arising from any reliance placed on the content. Where the content includes any translated material, BMJ does not warrant the accuracy and reliability of the translations (including but not limited to local regulations, clinical guidelines, terminology, drug names and drug dosages), and is not responsible for any error and/or omissions arising from translation and adaptation or otherwise.

Open access This is an open access article distributed in accordance with the Creative Commons Attribution Non Commercial (CC BY-NC 4.0) license, which permits others to distribute, remix, adapt, build upon this work non-commercially, and license their derivative works on different terms, provided the original work is properly cited, appropriate credit is given, any changes made indicated, and the use is non-commercial. See: http://creativecommons.org/licenses/by-nc/4.0/.

\section{ORCID iDs}

Anne-Marie Hill http://orcid.org/0000-0003-1411-6752

Rachael Moorin http://orcid.org/0000-0001-8742-7151

Max K Bulsara http://orcid.org/0000-0002-8033-6123

\section{REFERENCES}

1 Australian Bureau of Statistics. Disability, ageing and carers, Australia: summary of findings, 2018. Available: https://www.abs.gov. au/ausstats/abs@.nsf/mf/4430.0

2 World Health Organisation. Ageing and health, 2018. Available: https://www.who.int/news-room/fact-sheets/detail/ageing-andhealth

3 Ris I, Schnepp W, Mahrer Imhof R. An integrative review on family caregivers' involvement in care of home-dwelling elderly. Health Soc Care Community 2019;27:e95-111.

4 Carers UK. State of caring. A snapshot of unpaid care in the UK, 2019. Available: https://www.carersuk.org/news-and-campaigns/ state-of-caring-survey-2020

5 Carers NSW Australia. 40 years of carer surveys (1976-2016), 2014. Available: http://www.carersnsw.org.au/research/survey

6 Butterworth P, Pymont C, Rodgers B, et al. Factors that explain the poorer mental health of caregivers: results from a community survey of older Australians. Aust N Z J Psychiatry 2010;44:616-24.
7 Litzelman K, Skinner HG, Gangnon RE, et al. The relationship among caregiving characteristics, caregiver strain, and health-related quality of life: evidence from the survey of the health of Wisconsin. Qual Life Res 2015;24:1397-406.

8 Bom J, Bakx P, Schut F, et al. The impact of informal caregiving for older adults on the health of various types of caregivers: a systematic review. Gerontologist 2019;59:e629-42.

9 Betini RSD, Hirdes JP, Lero DS, et al. A longitudinal study looking at and beyond care recipient health as a predictor of long term care home admission. BMC Health Serv Res 2017;17:709.

10 Lopez Hartmann M, Wens J, Verhoeven V, et al. The effect of caregiver support interventions for informal caregivers of communitydwelling frail elderly: a systematic review. Int J Integr Care 2012;12:e133.

11 Toye C, Parsons R, Slatyer S, et al. Outcomes for family carers of a nurse-delivered hospital discharge intervention for older people (the further enabling care at home program): single blind randomised controlled trial. Int J Nurs Stud 2016;64:32-41.

12 Petrovic M, Gaggioli A. Digital mental health tools for caregivers of older adults-a scoping review. Front. Public Health 2020;8:128.

13 Hendrix CC, Matters D, Griffin T, et al. Academic-practice partnership for caregiver training and support: the Duke elder family/caregiver training (DEFT) center. N C Med J 2020;81:221-7.

14 Pritchard E, Cussen A, Delafosse V, et al. Interventions supporting caregiver readiness when caring for patients with dementia following discharge home: a mixed-methods systematic review. Australas J Ageing 2020;39.

15 Smith TO, Pearson M, Pfeiffer K, et al. Caregiver interventions for adults discharged from the hospital: systematic review and MetaAnalysis. J Am Geriatr Soc 2019;67:1960-9.

16 Corry M, Neenan K, Brabyn S, et al. Telephone interventions, delivered by healthcare professionals, for providing education and psychosocial support for informal caregivers of adults with diagnosed illnesses. Cochrane Database Syst Rev 2019;21.

17 Hill AM, Etherton-Beer C, McPhail S. Falls after hospital discharge: a randomised clinical trial of individualised multimodal falls prevention education. J Gerontol A Biol Sci Med Sci 2019;2019:1511-7.

18 Gill TM, Gahbauer EA, Murphy TE. Risk factors and precipitants of long-term disability in community mobility: a cohort study of older persons. Ann Intern Med 2012;156:131-40.

19 Jencks SF, Williams MV, Coleman EA. Rehospitalizations among patients in the Medicare fee-for-service program. N Engl J Med 2009;360:1418-28.

20 Hestevik CH, Molin M, Debesay J, et al. Older patients' and their family caregivers' perceptions of food, meals and nutritional care in the transition between hospital and home care: a qualitative study. BMC Nutr 2020;6:11.

21 Greysen SR, Harrison JD, Kripalani S, et al. Understanding patientcentred readmission factors: a multi-site, mixed-methods study. BM Qual Saf 2017;26:33-41.

22 Lilleheie I, Debesay J, Bye A, et al. The tension between carrying a burden and feeling like a burden: a qualitative study of informal caregivers' and care recipients' experiences after patient discharge from hospital. Int J Qual Stud Health Well-being 2021;16:1855751.

23 Allen Watts K, Malone E, Dionne-Odom JN, et al. Can you hear me now?: improving palliative care access through telehealth. Res Nurs Health 2021;44:226-37.

24 Wilz G, Soellner R. Evaluation of a short-term telephone-based cognitive behavioral intervention for dementia family caregivers. Clin Gerontol 2016;39:25-47.

25 Bakas T, McCarthy MJ, Israel J, et al. Adapting the telephone assessment and skill-building kit to the telehealth technology preferences of stroke family caregivers. Res Nurs Health 2021;44:81-91.

26 Waller A, Dilworth S, Mansfield E, et al. Computer and telephone delivered interventions to support caregivers of people with dementia: a systematic review of research output and quality. BMC Geriatr 2017;17:265.

27 Aoun SM, Stegmann R, Slatyer S, et al. Hospital postdischarge intervention trialled with family caregivers of older people in Western Australia: potential translation into practice. BMJ Open 2018;8:e022747.

28 Australian Commission on Safety and Quality in Health Care. Patient reported outcome measures, 2021. Available: https://www. safetyandquality.gov.au/our-work/indicators-measurement-andreporting/patient-reported-outcome-measures

29 Moher D, Hopewell S, Schulz KF, et al. CONSORT 2010 explanation and elaboration: updated guidelines for reporting parallel group randomised trials. J Clin Epidemiol 2010;63:e1-37. 
30 Chan A-W, Tetzlaff JM, Altman DG, et al. Spirit 2013 statement: defining standard protocol items for clinical trials. Ann Intern Med 2013;158:200-7.

31 Aggar C, Ronaldson S, Cameron ID. Self-Esteem in carers of frail older people: resentment predicts anxiety and depression. Aging Ment Health 2011;15:671-8.

32 Hoffmann TC, Glasziou PP, Boutron I, et al. Better reporting of interventions: template for intervention description and replication (TIDieR) checklist and guide. BMJ 2014;348:g1687.

33 Malouff J, Thorsteinsson E, Schutte N. The efficacy of problem solving therapy in reducing mental and physical health problems: a meta-analysis. Clin Psychol Rev 2007;27:46-57.

34 Ewing G, Austin L, Diffin J, et al. Developing a person-centred approach to carer assessment and support. Br J Community Nurs 2015;20:580-4.

35 CSNAT. The carer support needs assessment: a toolkit for practitioners. carer support needs assessment tool (CSNAT), 2015. Available: http://csnat.org/

36 Richardson J, lezzi A, Khan MA, et al. Validity and reliability of the assessment of quality of life (AQoL)-8D multi-attribute utility instrument. Patient 2014;7:85-96.

37 Richardson J, Khan MA, lezzi A, et al. Comparing and explaining differences in the magnitude, content, and sensitivity of utilities predicted by the EQ-5D, SF-6D, HUI 3, 15D, QWB, and AQoL-8D multiattribute utility instruments. Med Decis Making 2015;35:276-91.

38 Richardson J, lezzi A, Khan MA. Why do multi-attribute utility instruments produce different utilities: the relative importance of the descriptive systems, scale and 'micro-utility' effects. Quality of Life Research 2015;24:2045-53.

39 Maxwell A, Özmen M, lezzi A, et al. Deriving population norms for the AQoL-6D and AQoL-8D multi-attribute utility instruments from webbased data. Qual Life Res 2016;25:3209-19.

40 Archbold PG, Stewart BJ, Greenlick MR, et al. Mutuality and preparedness as predictors of caregiver role strain. Res Nurs Health 1990;13:375-84

41 Schumacher KL, Stewart BJ, Archbold PG, et al. Effects of caregiving demand, mutuality, and preparedness on family caregiver outcomes during cancer treatment. Oncol Nurs Forum 2008;35:49-56.
42 Henriksson A, Andershed B, Benzein E, et al. Adaptation and psychometric evaluation of the preparedness for caregiving scale, caregiver competence scale and rewards of caregiving scale in a sample of Swedish family members of patients with life-threatening illness. Palliat Med 2012;26:930-8.

43 Merluzzi TV, Philip EJ, Vachon DO, et al. Assessment of self-efficacy for caregiving: the critical role of self-care in caregiver stress and burden. Pall Supp Care 2011;9:15-24.

44 Cooper B, Kinsella GJ, Picton C. Development and initial validation of a family appraisal of caregiving questionnaire for palliative care. Psychooncology 2006;15:613-22.

45 Mahoney FI, Barthel DW. Functional evaluation: the BARTHEL index. Md State Med J 1965;14:61-5.

46 Aoun SM, Monterosso L, Kristjanson LJ, et al. Measuring symptom distress in palliative care: psychometric properties of the symptom assessment scale (SAS). J Palliat Med 2011;14:315-21.

47 Toye $\mathrm{C}$, Walker $\mathrm{H}$, Kristjanson LJ, et al. Measuring symptom distress among frail elders capable of providing self reports. Nurs Health Sci 2005;7:184-91.

48 Sterne JAC, White IR, Carlin JB, et al. Multiple imputation for missing data in epidemiological and clinical research: potential and pitfalls. BMJ 2009;338:2393.

49 Hawthorne G, Osborne R. Population norms and meaningful differences for the assessment of quality of life (AQoL) measure. Aust N Z J Public Health 2005;29:136-42.

50 Ware J, Kosinski M, Keller SD. A 12-Item short-form health survey: construction of scales and preliminary tests of reliability and validity. Med Care 1996;34:220-33.

51 Faul F, Erdfelder E, Buchner A, et al. Statistical power analyses using $\mathrm{G}^{\star}$ Power 3.1: tests for correlation and regression analyses. Behav Res Methods 2009;41:1149-60.

52 Moore GF, Audrey S, Barker M, et al. Process evaluation of complex interventions: medical Research Council guidance. BMJ 2015;350:h1258.

53 Braun V, Clarke V. Successful qualitative research; a practical guide for beginners. London: Sage Publications, 2013. 